\title{
Facteurs déterminants l'adoption des mesures de gestion intégrée de la fertilité des sols (GIFS) au nord du Bénin : une application du modèle probit multivarié au cas de producteurs de maïs
}

\author{
Souleïmane A. ADEKAMBI ${ }^{1 *}$, Jean Eudes A. CODJOVI ${ }^{2}$ et Jacob A. YABI ${ }^{3}$ \\ ${ }^{1}$ Institut Universitaire de Technologie (IUT), Centre de Recherche en Entrepreneuriat-Création et Innovation \\ (CRECI) - Laboratoire de Recherche en Dynamique Economie et Sociale (LARDES - UP), Université de \\ Parakou, Benin, B.P. 123, Parakou, Bénin. \\ ${ }^{2}$ Laboratoire d'Analyse et de Recherche sur les Dynamique Economiques et Sociales (LARDES), Université de \\ Parakou, Bénin. \\ ${ }^{3}$ Faculté d'Agronomie, Université de Parakou, Laboratoire d'Analyse et de Recherche sur les Dynamique \\ Economiques et Sociales (LARDES), Bénin. \\ *Auteur correspondant ; E-mail : adeksoul@gmail.com
}

\author{
Received: 23-01-2021
}

Accepted: 26-04-2021

Published: 30-04-2021

\section{RESUME}

La baisse du niveau de fertilité des terres cultivables du fait des pratiques agricoles inadaptées, de la déforestation et du changement climatique reste l'une des contraintes majeures de l'agriculture béninoise. Les mesures de Gestion Intégrée de la Fertilité des Sols (GIFS) en sont une des approches de gestion durales des terres à l'échelle des exploitations agricoles. Ainsi, l'étude a fait usage d'un modèle probit multivarié pour identifier les déterminants de l'adoption des mesures de GIFS au sein des exploitations agricoles productrices de maïs au Nord-Bénin. Elle a été conduite sur un échantillon de 100 producteurs de maïs sélectionnés suivant un sondage aléatoire stratifié. Les résultats ont révélé l'existence d'une interdépendance et de complémentarités dans l'adoption des différentes mesures de GIFS. Aussi, la majorité des producteurs de maïs enquêtés préfèrent adopter une combinaison de deux mesures de GIFS (32\%) ou de trois mesures (26\%). Par ailleurs, la distance du village au champ, l'appartenance à un groupement, le contact avec la vulgarisation, la superficie emblavée en maïs, la taille du cheptel et le nombre d'actifs agricoles restent les principaux facteurs déterminants l'adoption de ces mesures de GIFS par les producteurs.

(C) 2021 International Formulae Group. All rights reserved.

Mots clés : Bénin, changement climatique, gestion durable des terres, Gestion Intégrée de la Fertilité des Sols, pratiques agricoles, probit multivarié.

\section{Adoption of integrated soil fertility management measures in northern Benin: an application of the multivariate probit model to the case of maize producers}

\begin{abstract}
The decline in the level of fertility of arable land due to unsuitable agricultural practices, deforestation and climate change remains one of the major constraints of agriculture in Benin. Integrated Soil Fertility Management measures (GIFS) are one of the sustainable land management approaches that have been promoted at the level of farmers' communities. Thus, the study made use of a multivariate probit model to
\end{abstract}


identify the determinants of the adoption of GIFS measures within maize-producing farms in North Benin. It was carried out on a sample of 100 maize farmers selected following a stratified random survey. The results revealed the existence of interdependence and complementarities in the adoption of different GIFS measures. The majority of maize producers surveyed prefer to adopt a combination of two (32\%) or three GIFS measures (26\%). In addition, the distance from the village to the field, the membership of a group, the contact with the extension service, the area sown with maize, the size of the herd and the number of agricultural workers were identified as the important factors determining the adoption of these GIFS methods by producers.

(C) 2021 International Formulae Group. All rights reserved.

Keywords: Benin, climate change, sustainable land management, Integrated Soil Fertility Management measures, agricultural practices, multivariate probit.

\section{INTRODUCTION}

L'agriculture apparait aujourd'hui comme le secteur primordial pour régler la problématique d'insécurité alimentaire surtout dans les pays en voie de développement où la population connait un essor démographique important (Ramade, 2014). Elle constitue également l'un des secteurs les plus importants de l'économie aussi bien du point de vue de l'effectif de la population impliquée que de sa contribution au Produit Intérieur Brute (PIB).

$\mathrm{Au}$ Benin, l'agriculture contribue à environ $33 \%$ du PIB, fournit environ $75 \%$ des recettes d'exportation et occupe environ $70 \%$ de la population active (PSDSA, 2017). Toutefois, cette agriculture est de plus en plus confrontée à la dégradation progressive et continuelle de sa ressource capitale, la terre, sous l'effet de pratiques agricoles inadaptées, de la déforestation et du changement climatique (ProSol, 2016a). La dégradation des sols touche déjà plus des deux tiers des surfaces cultivées. Selon une étude de ProSol (2016b), en 10 ans, environ 2,2 millions d'hectares de terres agricoles se sont dégradées, soit $19 \%$ du territoire national. Aussi, $21 \%$ de la superficie de forêts a-t-elle été détruite en 10 ans au profit des terres agricoles et des habitations.

Cette dégradation engendre une baisse drastique de la quantité de nutriments et de matières organiques dans les sols, conduisant ainsi à l'appauvrissement de la fertilité de ces sols (FAO, 2015). Par exemple, les études menées par le laboratoire des sols de l'INRAB/LSSSE en 2016 dans les départements du Zou, Borgou et de l'Alibori au Bénin ont révélé que près de $90 \%$ des terres dans ces départements présentent des niveaux de fertilité généralement faibles. De telle baisse continuelle de la fertilité des sols a des effets néfastes sur la productivité agricole, la sécurité alimentaire des ménages et la pauvreté rurale (Adegbola et Adekambi, 2006). De ce fait, une inaction des divers acteurs concernés conduirait à un ralentissement du développement agricole des pays en voie de développement et une réelle menace à la stabilité alimentaire. Subséquemment, les terres sont donc au cœur de grands enjeux planétaires et constituent un élément indispensable du secteur agricole qu'il faut protéger et utiliser durablement (Montaigne et al., 2018).

Pour limiter cette dégradation croissante des sols, réhabiliter les sols dégradés et contribuer ainsi à améliorer la productivité des cultures, plusieurs approches de gestion durables des terres à l'échelle des exploitations agricoles ont été promues. Au Benin, les mesures de la Gestion Intégrée de la fertilité des Sols (GIFS) restent les plus vulgarisées par les services de conseils agricoles et les différents projets et programmes de protection de l'environnement. $\mathrm{Au}$ nombre des récents projets ayant contribué à la vulgarisation de ces mesures se trouve le projet de protection et de la réhabilitation des sols (ProSol) de l'initiative spéciale «un seul monde sans faim» de la GIZ, mis en œuvre dans 18 communes des départements du Zou, Borgou et de l'Alibori. Ces mesures de GIFS concernent l'utilisation des plantes améliorantes (Mucuna spp, Aeschinomenea, etc.), des légumineuses pérennes (Leucaena leucocephala, Gliricidia sepium, Acacia 
auriculiformis, Cassia siamea, etc.), des résidus de récoltes, la pratique de la rotation des cultures, du compostage et du micro fertilisants. Plusieurs études techniques aussi bien en station sous contrôle des chercheurs qu'en milieu paysan ont montré la durabilité de ces mesures (production du bois, des fourrages et aliments, tout en restaurant la fertilité) et leur impact positif et significatif sur la productivité des cultures (Azontondé, 1993 ; Akakpo et al., 1998 ; Etèka, 1998).

En dépit de leurs performances, ces mesures de GIFS sont faiblement adoptées (Adegbola et Adekambi, 2006; Yabi et al., 2016). A cet effet, des études récentes ont essayé d'élucider les facteurs qui déterminent l'adoption de ces pratiques de GIFS (Adegbola et Adekambi, 2006; Yabi et al., 2016; Issoufou et al., 2017). Par exemple, Yabi et al. (2016) dans leur étude, se sont intéressés aux fumures minérales et aux pratiques de lutte antiérosive, de rotation et d'association de cultures appropriées et ont montré, à travers un modèle logit binaire estimé par pratique de GIFS, que l'adoption de ces différentes pratiques dépend significativement du sexe du producteur, de son âge, de son niveau d'instruction, de la taille de son ménage, du mode de faire-valoir des terres mises en culture, de son appartenance à un groupement, de son accès aux technologies mises en évaluation et de son statut social dans la société. Généralement ces études empiriques ont eu à analyser les facteurs déterminants l'adoption des pratiques de GIFS en considérant que l'adoption de l'une de ces pratiques est indépendante de celle des autres prises individuellement en faisant usage des méthodes d'estimation conventionnelles basées sur le logit ou probit. Toutefois, de récentes études ont démontré l'existence d'une certaine corrélation significative entre certaines technologies de conservation des sols (Sileshi et al., 2019). Ainsi, le producteur pourrait adopter ou pas une pratique GIFS du fait de l'adoption ou non d'autres pratiques. La présente étude s'intéresse alors à identifier les déterminants de l'adoption des mesures de GIFS au sein des exploitations agricoles en tenant compte de l'interdépendance dans l'adoption des pratiques de GIFS par les producteurs.

\section{MATERIEL ET METHODES}

Approches théorique et empirique de modélisation de l'adoption des pratiques GIFS

La théorie sur l'adoption des innovations agricoles stipule que l'adoption reste une décision individuelle (Rogers, 2003) et se fonde ordinairement sur le principe économique de rationalité de la théorie néoclassique (Varian, 2006). Ainsi, le producteur adopte une nouvelle technologie si et seulement si cette dernière lui permet de maximiser son utilité. Le producteur adoptera une pratique de GIFS, la régénération du sol avec les légumineux par exemple, si l'utilité espérée, représentée par $U_{1}(\pi)$, est supérieure à celle qu'il obtiendrait s'il ne l'avait pas adoptée, représentée par $\mathrm{U}_{0}(\pi)$, soit $\mathrm{U}_{1}(\pi)>\mathrm{U}_{0}(\pi)$. Toutefois, l'utilité que le producteur obtient de l'adoption de l'une ou l'autre des pratiques de GIFS n'est pas observable. Elle dépend néanmoins des caractéristiques socioéconomiques et démographiques dudit producteur et de son environnement institutionnel $\left(X_{i}\right)$ et peut être donc représentée par la variable latente comme suit :

$U_{i}^{*}=X_{i} \beta+\varepsilon_{i}, i=1,2, \ldots, N$

Avec $\beta$ le vecteur de paramètres à estimer et $\varepsilon_{i}$ la perturbation aléatoire

Les approches analytiques les plus souvent utilisées dans les études de décision portant sur le choix des innovations pour estimer l'équation 1 sont le Probit et le Logit. Lorsque ce choix concerne une seule innovation, donc une variable dépendante dichotomique (l'adoption (qui pourrait être codé 1) ou non (qui pourrait être codé 0 ) d'une seule pratique agricole d'amélioration de la fertilité de sols), un modèle Logit ou Probit univarié est généralement utilisé (Oude Lansink et al., 2003). Par contre lorsque le choix devrait s'opérer entre plusieurs alternatives d'innovations (par exemple 
choisir entre plusieurs pratiques agricoles d'amélioration de la fertilité de sols) et de pratiques traditionnelles, la littérature conseille entre les modèles Logit ou Probit multinomiaux ou multivariés. Les modèles multinomiaux se basent sur l'indépendance des alternatives non pertinentes, c'est-à-dire que les termes d'erreur des équations de choix des alternatives s'excluent mutuellement (Greene et Hensher, 2003). Toutefois, les choix parmi les types de mesures GIFS ne s'excluent pas mutuellement; le producteur pourrait adopter une pratique donnée du fait d'une autre pratique qu'il utilise déjà ou envisage de pratiquer. De ce fait, les termes d'erreur aléatoire des différentes équations d'adoption des pratiques de GIFS peuvent donc être corrélés. Dans de pareilles circonstances, l'estimation des modèles Logit ou Probit multinomiaux conduirait à des estimateurs biaisés (Greene, 2008).

La présente étude s'est intéressée à quatre pratiques de GIFS que sont l'assolement et rotation, l'utilisation des matières organiques, les résidus de récolte et la régénération du sol avec les légumineuses. Comme argumenté plus haut, les producteurs ont tendance à adopter plusieurs pratiques de GIFS à la fois dans le but de faire face aux effets néfastes des problèmes de dégradation et de la baisse du niveau de fertilité des sols. De ce fait et partant de la littérature empirique sur l'adoption des technologies agricoles (Kassie et al., 2015), toutes les innovations complémentaires en termes d'utilités qu'elles permettent au producteur de gagner et de maximiser seront adoptées par ce dernier. Ce qui stipule une interdépendance des décisions $\mathrm{du}$ producteur d'adopter chacune de ces technologies agricoles. En d'autres termes, la décision d'adopter la pratique de GIFS $\mathrm{j}$ par le producteur i dépendrait de la décision d'adopter la pratique de GIFS $\mathrm{k}$, ainsi de suite. Lorsque l'interdépendance dans les décisions d'adoption des technologies agricoles est soupçonnée, la littérature conseille l'utilisation d'un modèle de régression probit multivarié pour une estimation sans biais des estimateurs (Wu et Babcock, 1998 ; Timu et al., 2014). Le probit multivarié est une extension du modèle probit bivarié qui use des techniques de simulation de Monte Carlo pour estimer simultanément le système d'équations de régression probit multivarié (Greene, 2008). Les adoptions simultanées pour les quatre pratiques de GIFS peuvent être modélisées par un système de quatre équations d'adoption dichotomiques $Y_{i}$ comme suit :

$\left\{\begin{array}{l}Y_{1}=1 \text { si } U_{1}^{*}>U_{0}^{*}, Y_{1}=0 \text { si autrement } \\ Y_{2}=1 \text { si } U_{2}^{*}>U_{0}^{*}, Y_{2}=0 \text { si autrement } \\ Y_{3}=1 \text { si } U_{3}^{*}>U_{0}^{*}, Y_{3}=0 \text { si autrement } \\ Y_{4}=1 \text { si } U_{4}^{*}>U_{0}^{*}, Y_{4}=0 \text { si autrement }\end{array}\right.$ (2)

Le modèle de régression probit multivarié a été adopté pour estimer la probabilité d'adoption des pratiques de GIFS (équation 2) dans le but de tenir compte d'éventuelle corrélation entre les termes d'erreurs des différentes équations binaires d'adoption (Greene, 2008). Le modèle probit multivarié a déjà été utilisé dans un certain nombre d'études empiriques évaluant les facteurs qui influent l'adoption simultanée de plusieurs technologies agricoles (DassoundoAssogba et al., 2019). Le modèle empirique estimé avec les variables inclues dans les estimations se présente de la façon suivante :

$$
\begin{aligned}
& \text { GIFS } S_{j}=a_{1} \text { sexe }_{i}+a_{1} \text { exper }+a_{1} \text { distril } c_{i}+a_{1} \text { alphab } b_{i}+a_{1} \text { actifa } a_{i}+a_{1} \text { group } \\
& +a_{1} \text { cullgar }_{i}+a_{1} \text { supm }_{i}+a_{1} \text { percepfertil }{ }_{i}+a_{1} \text { tcheptel } l_{i}+\varepsilon_{i}
\end{aligned}
$$

Avec GIFSj l'ensemble des variables dépendantes que sont l'assolement et rotation, l'utilisation des matières organiques, les résidus de récolte et la régénération du sol avec les légumineuses. Chaque variable dépendante de l'équation (3) est une variable binaire qui prend la valeur 1 si le producteur i adopte la pratique de GIFS $\mathrm{j}$ (avec $\mathrm{j}=$ assolement et rotation, matières organiques, résidus de récolte et régénération du sol avec les légumineuses.) et 0 si non. Les différentes variables indépendantes utilisées dans l'estimation du modèle probit multivarié sont décrites dans le Tableau 1.

\section{Choix de la zone d'étude}

La présente étude a été conduite au Nord du Bénin, où la production du maïs a connu une évolution spectaculaire ces deux 
dernières décennies et est désormais perçue comme une culture de rente (Degla et al., 2020). Par ailleurs, la commune de Bembèrèkè a été plus précisément retenue dans le cadre de cette étude (Figure 1) car il s'agit de l'une des communes du département de Borgou ayant bénéficié au cours des cinq dernières années avant les enquêtes de la présente étude des interventions du projet ProSol sur les mesures GIFS. Le choix des villages d'enquêtes a été fait de manière raisonnée et avec l'aide des techniciens de terrain du ProSol et tenant compte de l'intensité de la problématique relative à la fertilité des sols. Au total trois villages d'intervention du projet ProSol de la commune de Bembèrèkè ont été sélectionnés à savoir : Béroubouay, Gamia et Ina.

\section{Unité de base de la recherche et échantillonnage}

L'unité de recherche est constituée des chefs d'exploitations producteurs du maïs des villages retenus. Ces producteurs du maïs à l'intérieur de chaque village ont été sélectionnés de manière aléatoire. En effet, en séance tenante, la liste exhaustive des producteurs de maïs utilisateurs d'au moins une mesure GIFS a été établie et numérotée de 1 à $\mathrm{n}$ avec l'aide du technicien du ProSol d'une part et celle des producteurs qui n'appliquent aucune de ces mesures GIFS a été également établie. Ces deux listes ont été ensuite saisies et soumises séparément à la fonction de sélection aléatoire de EXCEL. Au total, les données ont été collectées auprès d'un échantillon aléatoire stratifié constitué de 100 producteurs de maïs dans la zone d'étude, à raison de 50 adoptants et 50 non adoptants. La répartition des producteurs enquêtés par village se présente dans le Tableau 2. Les données collectées sont aussi bien quantitatives que qualitatives. Elles sont relatives à la campagne agricole 2019-2020 et concernent notamment les caractéristiques socio-économiques et démographiques des chefs d'exploitations enquêtés (sexe, niveau d'instruction, taille du ménage et nombre d'actifs agricoles, contact avec les structures de vulgarisation, activité principale, appartenance à un groupement, etc.), les mesures de GIFS appliquées et les données relatives à la perception des producteurs.

Tableau 1 : Description des variables indépendantes incluses dans les modèles estimés.

\begin{tabular}{lll}
\hline Variables & Description & Modalités \\
\hline Sexe & Sexe & Variable binaire $(1=$ homme, $0=$ femme $)$ \\
Exper & Expérience en agriculture & Variable continue \\
Distvilc & Distance village champs & Variable continue \\
Alphab & Alphabétisation & Variable binaire $(1=$ Oui, $0=$ Non $)$ \\
Actifa & Actifs agricoles & Variable continue \\
Group & Appartenance à un groupement & Variable binaire $(1=$ Oui, $0=$ Non $)$ \\
Cvulgar & Contact avec la vulgarisation & Variable binaire $(1=$ Oui, $0=$ Non $)$ \\
Supm & Superficie du maïs & Variable continue \\
Percepfertil & Perception sur le niveau de fertilité & Variable binaire $(1=$ peu fertile, $0=$ non $)$ \\
Tcheptel & des sols & Variable continue \\
\hline
\end{tabular}




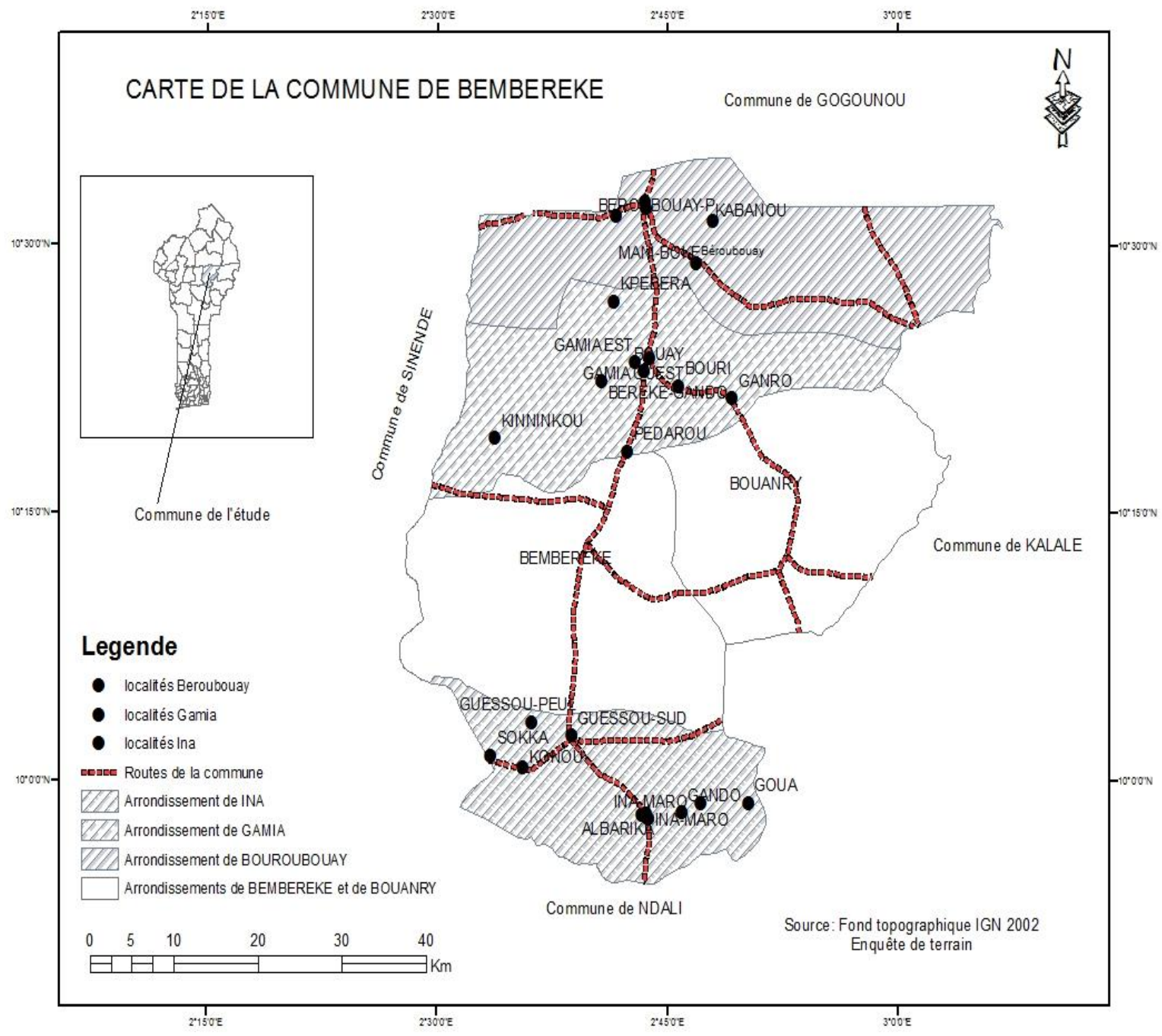

Figure 1 : Carte de la commune de Bembérékè.

Source : Auteurs, 2020.

Tableau 2: Répartition des enquêtés par village.

\begin{tabular}{lccc}
\hline Villages & $\begin{array}{c}\text { Adoptants d'au moins une } \\
\text { mesure de GIFS }\end{array}$ & $\begin{array}{c}\text { Non adoptants de } \\
\text { mesures de GIFS }\end{array}$ & Ensemble \\
\hline Béroubouay & 15 & 15 & $\mathbf{3 0}$ \\
Gamia & 15 & 15 & $\mathbf{3 0}$ \\
Ina & 20 & 20 & $\mathbf{4 0}$ \\
\hline Total & $\mathbf{5 0}$ & $\mathbf{5 0}$ & $\mathbf{1 0 0}$ \\
\hline
\end{tabular}




\section{RESULTATS}

\section{Caractéristiques sociodémographiques et économiques des enquêtés}

Le Tableau 3 présente les caractéristiques sociodémographiques et économiques des producteurs de maïs enquêtés suivant leur statut d'adoptant d'au moins une des quatre pratiques de GIFS étudiées à savoir: l'assolement et rotation, l'utilisation des matières organiques, les résidus de récolte et la régénération du sol avec les légumineuses. Les variables telles que le sexe, le niveau d'instruction, le nombre moyen d'actifs agricoles par ménage, le nombre d'années d'expérience dans la production agricole, l'activité principale ainsi que la principale culture produite, le niveau moyen de revenu, la distance par rapport au marché le plus proche, le mode d'accès à la terre, la superficie disponible, la perception du niveau de fertilité des terres cultivées, l'accès au crédit, la pratique de l'élevage et la taille moyenne de cheptel par ménage ne varient pas de manière significative suivant le statut d'adoption.

Par contre, le niveau d'alphabétisation, l'âge des enquêtés, la taille du ménage, la superficie emblavée en maïs, le contact avec la vulgarisation et le nombre moyen de visites par an varient significativement suivant le statut d'adoption. Environ $27 \%$ des producteurs de maïs enquêtés sont alphabétisés avec une prédominance des adoptants de GIFS. En moyenne, les adoptants de GIFS sont plus âgés que les non-adoptants (46 ans contre 41 ans pour les non-adoptants). La taille de ménages des adoptants d'au moins une des quatre pratiques de GIFS étudiées est significativement plus élevée que celle des non-adoptants (11 personnes en moyenne contre 9 pour les non-adoptants). Les adoptants de GIFS emblavent en moyenne 5,38 ha de maïs contre 3,28 ha pour les nonadoptants. La proportion des adoptants ayant de contact avec la vulgarisation est plus élevée (70\% des adoptants) que celle des nonadoptants (30\%). Aussi, les adoptants sont-ils plus visités par les agents d'encadrement que les non-adoptants (4 visites en moyenne par an contre 3 pour les non-adoptants).
Analyse du taux d'adoption des mesures de la gestion intégrée de la fertilité des sols

Le Tableau 4 présente les taux d'adoption des différentes mesures de GIFS par les producteurs. Les résultats révèlent que la régénération du sol avec les légumineuses et les résidus de récoltes restent les mesures de GIFS les plus pratiquées dans la zone d'étude (35\% et $34 \%$ des producteurs de maïs enquêtés respectivement). En ce qui concerne les mesures de GIFS telles que l'assolement et rotation et l'utilisation des fertilisants organiques, elles sont pratiquées respectivement par $21 \%$ et $28 \%$ des producteurs de maïs enquêtés. L'analyse de l'adoption combinée des différentes mesures de GIFS révèle que la majorité des adoptants de GIFS (32\%) ont pratiqué deux mesures GIFS à la fois. Aussi, environ 26\% des producteurs de maïs enquêtés ont-ils pratiqué trois mesures de GFIS à la fois contre $22 \%$ les quatre mesures de GIFS étudiées.

\section{Déterminants d'adoption des mesures GIFS}

Le Tableau 5 présente les résultats d'estimation du modèle probit multivarié. Le test $\mathrm{du}$ ratio de vraisemblance pour la corrélation globale des termes d'erreurs des différents modèles estimés (chi2 $(6)=21,21$; $\mathrm{p}<0,001)$ est significativement différent de zéro au seuil de $1 \%$ et permet donc de rejeter l'hypothèse de l'indépendance des choix des différentes mesures de GIFS. La décision d'adopter une mesure de GIFS est donc déterminée par celle d'une autre et vice-versa. La corrélation entre la décision d'adopter les résidus de récolte et la régénération des sols avec les légumineuses est positive et significative au seuil de $1 \%$ (rho $=0,774 ; \mathrm{p}<$ $0,01)$. Aussi, de ce tableau, il ressort que les variables qui influencent significativement l'adoption de l'une au moins des quatre mesures de GIFS sont : la distance du village au champ du producteur, l'appartenance à un groupement de producteurs, le contact avec la vulgarisation, la superficie emblavée en maïs, la taille du cheptel et le nombre d'actifs de l'exploitation. 
La distance entre le village et le champ principal de production de maïs du producteur influence négativement et significativement l'adoption des résidus de récolte au seuil de $1 \%(\alpha=-0,129 ; \mathrm{p}<0,01)$. Quant au contact avec les agents de la vulgarisation, il a un effet positif et significatif au seuil de $1 \%$ sur l'adoption de la régénération du sol avec les légumineuses $(\alpha=2,030 ; p<0,01)$. Il en est de même pour le nombre d'actifs agricoles qui présente un effet positif et significatif au seuil de $1 \%$ sur l'adoption de résidus de récolte $(\alpha$ $=0,152 ; \mathrm{p}<0,01)$. Le nombre d'actifs agricoles au sein du ménage étant un proxy du degré de disponibilité de la main-d'œuvre (familiale de manière spécifique), l'augmentation du nombre d'actifs du ménage accroît le niveau de disponibilité de la main d'œuvre familiale. En ce qui concerne l'appartenance à un groupement de producteurs, elle a un effet positif et significatif au seuil de $5 \%$ sur le choix des matières organiques $(\alpha=1,278 ; \mathrm{p}<0,05)$. La superficie du maïs a un effet négatif et significatifau seuil de $1 \%$ sur le choix des résidus de récolte comme stratégies de régénération de la fertilité des sols $(\alpha=$ $0,255, \mathrm{p}<0,01)$. Enfin, la taille du cheptel influence positivement et significativement l'adoption des fertilisants organiques (fumure et déjection animale) $(\alpha=0,068 ; \mathrm{p}<0,05)$ et d'assolement-rotation $(\alpha=0,050 ; \mathrm{p}<0,05)$ au seuil de $5 \%$.

Tableau 3 : Statistiques descriptives des variables suivant le statut d'adoption.

\begin{tabular}{|c|c|c|c|c|}
\hline Variables & Modalités & $\begin{array}{l}\text { Adoptants de } \\
\text { mesures de } \\
\text { GIFS }\end{array}$ & $\begin{array}{l}\text { Non-adoptants } \\
\text { de mesures de } \\
\text { GIFS }\end{array}$ & Test de comparaison \\
\hline \multirow{2}{*}{ Sexe } & Femme & $60,7 \%$ & $39,3 \%$ & \multirow[t]{2}{*}{ Sig. $($ Khi-Chi-deux $)=0,181$} \\
\hline & Homme & $45,8 \%$ & $54,2 \%$ & \\
\hline \multirow{5}{*}{ Ethnie } & Bariba & $37,5 \%$ & $62,5 \%$ & \multirow[t]{2}{*}{ Sig. $($ Khi-Chi-deux $)=0,075$} \\
\hline & Cotocoli & $0,0 \%$ & $100,0 \%$ & \\
\hline & Gando & $81,65 \%$ & $18,35 \%$ & \multirow{6}{*}{ Sig. $($ Khi-Chi-deux $)=0,222$} \\
\hline & Idaatcha & $0,0 \%$ & $100,0 \%$ & \\
\hline & Peulh & $60,0 \%$ & $40,0 \%$ & \\
\hline \multirow{3}{*}{$\begin{array}{l}\text { Situation } \\
\text { Matrimoniale }\end{array}$} & Célibataire & $0,0 \%$ & $100,0 \%$ & \\
\hline & Marié & $50,5 \%$ & $49,5 \%$ & \\
\hline & Veuf (ve) & $100,0 \%$ & $0,0 \%$ & \\
\hline \multicolumn{2}{|c|}{ Alphabétisation } & $66,7 \%$ & $33,3 \%$ & Sig. $($ Khi-Chi-deux $)=0,043$ \\
\hline \multicolumn{2}{|c|}{ Niveau d'instruction } & $35,0 \%$ & $65,0 \%$ & Sig. $($ Khi-Chi-deux $)=0,134$ \\
\hline \multicolumn{2}{|c|}{ Nombre d'années d'étude } & $7(+/-4)$ & $5(+/-4)$ & Sig. $($ test $t-$ Student $)=0,949$ \\
\hline \multicolumn{2}{|c|}{ Age de l'enquêté (an) } & $46(+/-12)$ & $41(+/-12)$ & Sig. $($ test $\mathrm{t}$-Student $)=0,034$ \\
\hline \multicolumn{2}{|c|}{ Taille du ménage } & $11(+/-6)$ & $9(+/-5)$ & Sig. $($ test $\mathrm{t}$-Student $)=0,032$ \\
\hline \multicolumn{2}{|l|}{ Actifs agricoles } & $4(+/-3)$ & $4(+/-3)$ & Sig. $($ test t-Student $)=0,741$ \\
\hline \multicolumn{2}{|c|}{$\begin{array}{l}\text { Expériences dans la production } \\
\text { agricole }\end{array}$} & $17(+/-10)$ & $14(+/-8)$ & Sig. $($ test $t$-Student $)=0,115$ \\
\hline \multirow{4}{*}{$\begin{array}{l}\text { Activité } \\
\text { principale }\end{array}$} & Agriculture & $53,3 \%$ & $46,7 \%$ & \multirow[t]{4}{*}{ Sig. $($ Khi-Chi-deux $)=0,145$} \\
\hline & Artisanat & $0,0 \%$ & $100,0 \%$ & \\
\hline & Commerçants & $25,0 \%$ & $75,0 \%$ & \\
\hline & Autres & $0,0 \%$ & $100,0 \%$ & \\
\hline \multirow{2}{*}{$\begin{array}{l}\text { Culture } \\
\text { principale }\end{array}$} & Arachide & $33,3 \%$ & $66,7 \%$ & \multirow[t]{2}{*}{ Sig. $($ Khi-Chi-deux $)=0,385$} \\
\hline & Coton & $20,0 \%$ & $80,0 \%$ & \\
\hline
\end{tabular}




\begin{tabular}{|c|c|c|c|c|}
\hline Variables & Modalités & $\begin{array}{l}\text { Adoptants de } \\
\text { mesures de } \\
\text { GIFS }\end{array}$ & $\begin{array}{l}\text { Non-adoptants } \\
\text { de mesures de } \\
\text { GIFS }\end{array}$ & Test de comparaison \\
\hline \multirow{9}{*}{$\begin{array}{l}\text { Revenu } \\
\text { agricole }\end{array}$} & Maïs & $52,8 \%$ & $47,2 \%$ & \multirow{9}{*}{ Sig. $($ Khi-Chi-deux $)=0,243$} \\
\hline & Manioc & $0,0 \%$ & $100,0 \%$ & \\
\hline & $\mathrm{Riz}$ & $0,0 \%$ & $100,0 \%$ & \\
\hline & Soja & $50,0 \%$ & $50,0 \%$ & \\
\hline & Sorgho & $100,0 \%$ & $0,0 \%$ & \\
\hline & $\begin{array}{l}\text { Inférieur à } \\
100.000\end{array}$ & $37,9 \%$ & $62,1 \%$ & \\
\hline & $\begin{array}{l}100.000- \\
200.000\end{array}$ & $58,8 \%$ & $41,2 \%$ & \\
\hline & $\begin{array}{l}200.000- \\
300.000\end{array}$ & $62,5 \%$ & $37,5 \%$ & \\
\hline & $\begin{array}{l}\text { Supérieur à } \\
300.000\end{array}$ & $42,9 \%$ & $57,1 \%$ & \\
\hline \multicolumn{2}{|c|}{$\begin{array}{l}\text { Part sur } 10 \text { du revenu du maïs } \\
\text { dans le revenu du ménage }\end{array}$} & $\begin{array}{c}6,92 \\
(+/-1,77)\end{array}$ & $\begin{array}{c}5,04 \\
(+/-2,43)\end{array}$ & Sig. $($ test $t-$ Student $)=0,121$ \\
\hline \multicolumn{2}{|c|}{ Distance village-champ } & $\begin{array}{c}2,70 \\
(+/-3,73)\end{array}$ & $\begin{array}{c}3,09 \\
(+/-4,375)\end{array}$ & Sig. $($ test $t$-Student $)=0,721$ \\
\hline \multicolumn{2}{|c|}{ Distance village-marché } & $\begin{array}{c}1,51 \\
(+/-1,13)\end{array}$ & $\begin{array}{c}1,12 \\
(+/-1,37)\end{array}$ & Sig. $($ test t-Student $)=0,111$ \\
\hline \multirow{5}{*}{$\begin{array}{l}\text { Mode de } \\
\text { faire valoir } \\
\text { de la terre }\end{array}$} & Héritage & $56,2 \%$ & $43,8 \%$ & \multirow[t]{5}{*}{ Sig. $($ Khi-Chi-deux $)=0,28$} \\
\hline & Location & $50,0 \%$ & $50,0 \%$ & \\
\hline & Don & $10,0 \%$ & $90,0 \%$ & \\
\hline & Emprunt & $60,0 \%$ & $40,0 \%$ & \\
\hline & Achat & $0,0 \%$ & $100,0 \%$ & \\
\hline \multirow{3}{*}{$\begin{array}{l}\text { Niveau de } \\
\text { Fertilité du } \\
\text { sol }\end{array}$} & Non fertile & $0,0 \%$ & $100,0 \%$ & \multirow[t]{3}{*}{ Sig. $($ Khi-Chi-deux $)=135$} \\
\hline & Peu Fertile & $50,0 \%$ & $50,0 \%$ & \\
\hline & Très Fertile & $100,0 \%$ & $0,0 \%$ & \\
\hline \multicolumn{2}{|c|}{ Superficie disponible } & $\begin{array}{c}11,78 \\
(+/-7,26)\end{array}$ & $\begin{array}{c}13,24 \\
(+/-7,82)\end{array}$ & Sig. $($ test $t-S t u d e n t)=0,335$ \\
\hline \multicolumn{2}{|c|}{ Superficie emblavée } & $\begin{array}{c}7,92 \\
(+/-3,10)\end{array}$ & $\begin{array}{c}8,42 \\
(+/-+/-3,76)\end{array}$ & Sig. $($ test $t-$ Student $)=0,470$ \\
\hline \multicolumn{2}{|c|}{ Superficie cultivée du maïs } & $\begin{array}{c}5,38 \\
(+/-5,39)\end{array}$ & $\begin{array}{c}3,28 \\
(+/-1,62)\end{array}$ & Sig. (test t-Student $)=0,010$ \\
\hline \multicolumn{2}{|c|}{ Appartenance à un groupement } & $60,0 \%$ & $40,0 \%$ & Sig. $($ Khi-Chi-deux $)=0,401$ \\
\hline \multicolumn{2}{|c|}{ Accès au crédit formel } & $48,0 \%$ & $52,0 \%$ & Sig. $($ Khi-Chi-deux $)=0,817$ \\
\hline \multicolumn{2}{|c|}{ Contact avec la vulgarisation } & $70,0 \%$ & $30,0 \%$ & Sig. $($ Khi-Chi-deux $)=0,000$ \\
\hline \multicolumn{2}{|c|}{$\begin{array}{l}\text { Nombre de visites des agents de } \\
\text { vulgarisation par an }\end{array}$} & $4(+/-3)$ & $3(+/-2)$ & Sig. $($ test $\mathrm{t}$-Student $)=0,041$ \\
\hline \multicolumn{2}{|c|}{ Pratique d'élevage } & $55,2 \%$ & $44,8 \%$ & Sig. $($ Khi-Chi-deux $)=0,137$ \\
\hline \multicolumn{2}{|c|}{ Taille du cheptel } & $6(+/-9)$ & $9(+/-15)$ & Sig. $($ test $t$-Student $)=0,230$ \\
\hline
\end{tabular}


Tableau4 : Taux d'adoption des mesures de GIFS par les producteurs.

\begin{tabular}{|c|c|c|c|c|c|c|c|c|}
\hline \multicolumn{5}{|l|}{ Mesures de GIFS } & \multicolumn{4}{|c|}{ Adoptants de mesures de GIFS (\%) } \\
\hline \multicolumn{5}{|l|}{ Assolement et rotation } & \multicolumn{3}{|c|}{21} & \\
\hline \multicolumn{5}{|l|}{ Résidu de récoltes } & \multicolumn{3}{|c|}{34} & \\
\hline \multicolumn{5}{|l|}{ Régénération du sol avec les légumineuses } & \multicolumn{3}{|c|}{35} & \\
\hline \multicolumn{5}{|l|}{ Fertilisants organiques } & \multicolumn{3}{|c|}{28} & \\
\hline \multicolumn{9}{|c|}{ Nombre de mesures GIFS adoptées à la fois } \\
\hline \multicolumn{5}{|l|}{ Une mesure } & \multicolumn{3}{|c|}{20} & \\
\hline \multicolumn{5}{|l|}{ Deux mesures } & \multicolumn{3}{|c|}{32} & \\
\hline \multicolumn{5}{|l|}{ Trois mesures } & \multicolumn{3}{|c|}{26} & \\
\hline \multicolumn{5}{|l|}{ Quatre mesures } & \multicolumn{3}{|c|}{22} & \\
\hline \multicolumn{9}{|l|}{ Source: Données enquête, Jan-Fév. 2020} \\
\hline \multicolumn{9}{|c|}{ Tableau 5 : Estimation du modèle Probit multivarié. } \\
\hline \multirow{2}{*}{ Variables } & \multicolumn{2}{|c|}{ Assolement et rotation } & \multicolumn{2}{|c|}{$\begin{array}{c}\text { Utilisation des matières } \\
\text { organiques }\end{array}$} & \multicolumn{2}{|c|}{ Résidus de récolte } & \multicolumn{2}{|c|}{$\begin{array}{c}\text { Régénération du sol avec } \\
\text { les légumineuses }\end{array}$} \\
\hline & Coefficients & Test z & Coefficients & Test z & Coefficients & Test $\mathrm{z}$ & Coefficients & Test z \\
\hline Sexe $(1=$ Homme et $0=$ Femme $)$ & $-0,080$ & $-0,250$ & 0,276 & 0,530 & $-0,445$ & $-1,350$ & $-0,676$ & $-1,700$ \\
\hline Expérience en agriculture (ans) & $-0,009$ & $-0,550$ & 0,000 & $-0,020$ & $-0,003$ & $-0,170$ & $-0,029$ & $-1,260$ \\
\hline Distance village champs $(\mathrm{km})$ & 0,076 & 1,780 & $-0,259$ & $-1,730$ & $-0,129 * * *$ & $-2,520$ & $-0,061$ & $-1,220$ \\
\hline $\begin{array}{l}\text { Alphabétisation ( } 1=\text { Oui, } 0 \text { si } \\
\text { non) }\end{array}$ & 0,051 & 0,150 & $-1,149$ & $-1,640$ & 0,151 & 0,450 & $-0,072$ & $-0,200$ \\
\hline
\end{tabular}


S. A. ADEKAMBI et al. / Int. J. Biol. Chem. Sci. 15(2): 664-678, 2021

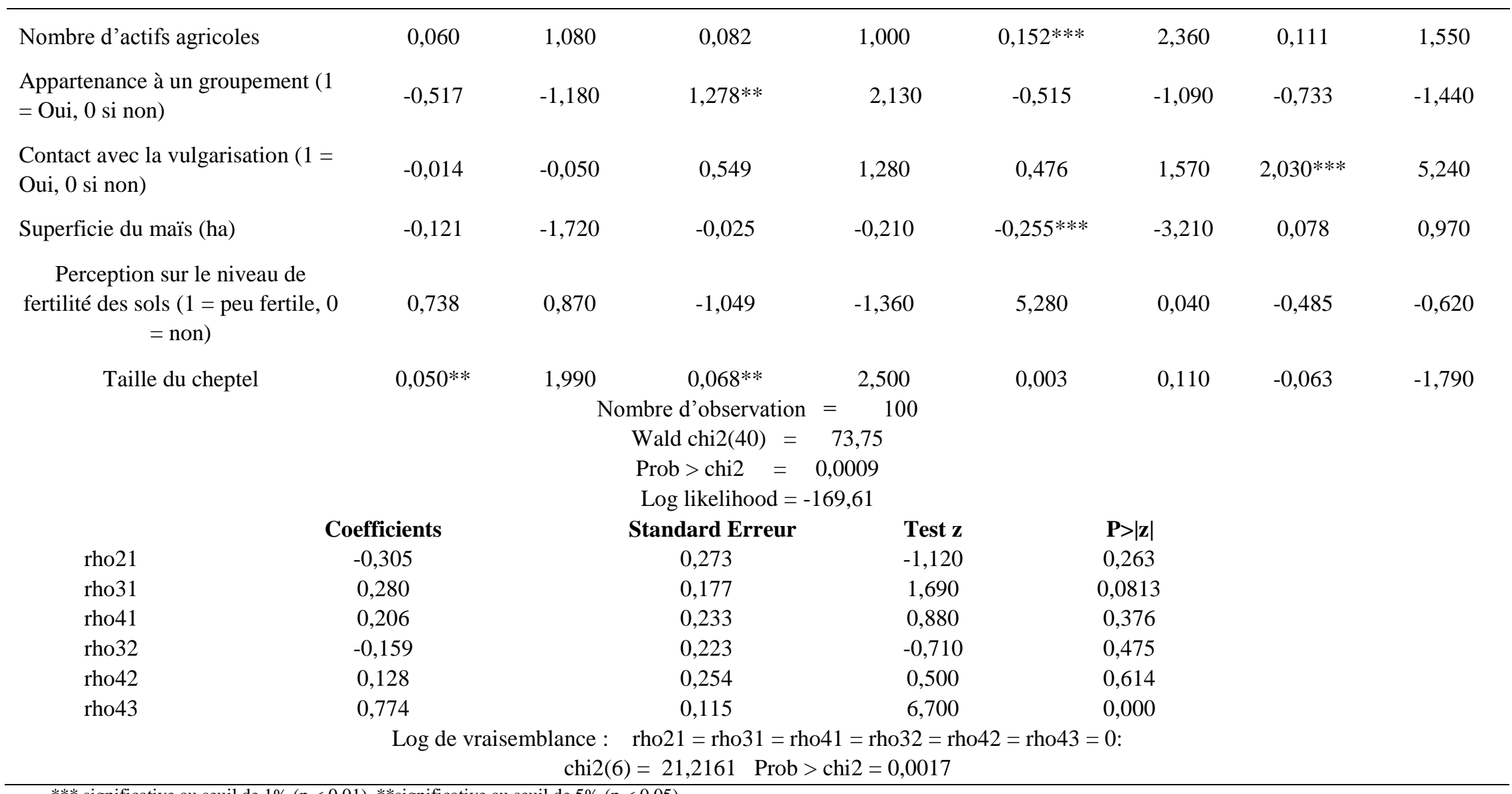

*** significative au seuil de $1 \%(\mathrm{p}<0,01)$, **significative au seuil de $5 \%(\mathrm{p}<0,05)$.

Source: Données enquête, Jan-Fév. 2020. 


\section{DISCUSSION}

L'augmentation de la production agricole est au cœur de toutes les discussions au sein des exploitations agricoles. A cet effet, les pratiques de GIFS sont de plus en plus adoptées par le producteurs comme des stratégies de régénération de la fertilité des sols. Ainsi, les résultats révèlent que la régénération du sol avec les légumineuses et les résidus de récoltes restent les mesures de GIFS les plus pratiquées dans la zone d'étude et que que la majorité des adoptants de GIFS ont pratiqué au moins deux mesures de GIFS à la fois. Ces résultats indiquent donc une complémentarité entre certaines mesures de GIFS adoptées par les producteurs dans la zone d'étude. La corrélation positive et significative observée suggère donc que l'adoption des " résidus de récolte » comme stratégie de gestion de la fertilité des sols s'accompagne de l'adoption de la " régénération avec les légumineuses ». Autrement dit, les producteurs de maïs qui adoptent les « résidus de récolte » comme mesure de GIFS pratiquent nécessairement en complément de la « régénération avec les légumineuses ». Ces deux mesures de GIFS restent la combinaison la plus adoptée par les producteurs de maïs de la zone d'étude. Ces résultats sont semblables à ceux d'autres auteurs qui ont aussi observé l'existence de corrélations significatives entre les technologies agricoles adoptées par les producteurs (ex. Danso-Abbeam et Baiyegunhi, 2017 ; Adekambi et Hinnou, 2020).

Par ailleurs, plusieurs facteurs infuencent l'adoption des mesures de GIFS. L'influence négative et significative de la distance entre le village et le champ principal de production de maïs du producteur implique que plus le champ principal de production de maïs du producteur est distant du village, plus faible est la probabilité d'adopter les résidus de récolte. Cela s'explique par le fait que les résidus de récolte des champs distants sont exposés aux dégâts des bœufs de transhumance par manque de surveillance du producteur. De pareil constat d'effet négatif de la distance d'habitation sur la décision d'adoption des producteurs est aussi fait dans plusieurs autres études empiriques (ex. Shiferaw et Tesfaye, 2005, Teferi et al., 2015). Le contact du producteur avec les agents des services techniques de conseils agricoles favorise l'adoption de la pratique de la régénération du sol avec les légumineuses. Cela peut s'expliquer par le fait que les structures de la vulgarisation fournissent aux producteurs les informations techniques nécessaires à l'adoption des innovations vulgarisées et les accompagnent tout au long de la phase d'essais desdites innovations. Ces résultats sont identiques à ceux de Mariano et al. (2012), Issoufou et al. (2017) et Adechian et al. (2020) qui ont tous observés des effets positifs et significatifs du contact des producteurs avec les agents du conseil agricole sur l'adoption des innovations agricoles.

Le nombre d'actifs agricoles de l'exploitation agricole présente un effet positif et significatif au seuil de $1 \%$ sur l'adoption des résidus de récolte $(\alpha=0,152 ; \mathrm{p}<0,01)$. De ce fait, plus la main-d'œuvre familiale au sein du ménage est disponible, plus grande est la probabilité d'adopter les résidus de récolte comme technique de GIFS. Ce qui veut dire qu'un nombre élevé d'actifs agricoles encourage l'adoption de cette mesure. Ces résultats corroborent les conclusions de Yabi et al. (2016) et de Kpadenou et al. (2019) selon lesquelles le nombre d'actifs agricoles a un effet positif sur l'adoption des technologies agricoles, surtout celles qui semblent être exigeantes en main-d'œuvre.

Les résultats ont également révélé que l'appartenance à un groupement favorise l'adoption des fertilisants organiques (fumure et déjection animale). Ces résultats s'expliquent par le fait que les groupements de producteurs constituent le plus souvent des centres d'informations, de vulgarisation, d'entraides sociales, et de partages d'expériences. Ces résultats sont conformes à ceux obtenus des études empiriques antérieures (ex. Kpadenou et al., 2019 et Kini et al., 2020). Ces derniers ont trouvé que les producteurs membres d'associations ont tendance à faire usage des semences certifiées agricoles et/ou adopter des pratiques biologiques de production agricoles. 
Toutefois, ces résultats sont contraires à ceux de Yabi et al. (2016) qui ont obtenu un effet significativement négatif de l'appartenance du producteur à un groupement sur sa décision d'adoption les technologies agricoles.

L'effet négatif et significatif de la variable «superficie emblavée en maïs » sur le choix des résidus de récolte signifierait que plus grande est la superficie cultivée en maïs, moins est la probabilité d'adoption des résidus de récolte comme stratégies de régénération de la fertilité des sols. En effet, l'exploitation des résidus de récolte consiste en de restitutions organiques (enfouissement des résidus de récolte) aux sols pour la gestion de fertilité des sols. La contrainte majeure reste la disponibilité de la main-d'œuvre. La faible adoption des résidus de récolte comme stratégies de régénération de la fertilité des sols pour les producteurs emblavant de grande superficies de maïs s'expliquerait donc par l'exigence en main-d'œuvre que requiert l'enfouissement de ces résidus. Ces résultats sont semblables à ceux de Sanou et Soule (2017) qui ont montré que l'adoption de la technique d'enfouissement de la paille du riz est limitée du fait de son exigeante en main d'œuvre abondante.

Enfin, la possession d'un cheptel important faciliterait l'adoption des fertilisants organiques et la pratique d'assolementrotation. La facilitation de l'adoption des fertilisants organiques pourrait s'expliquer par le fait que la possession d'un cheptel important permet au producteur de restituer directement au champ les déjections animales par le parcage direct des animaux sur les parcelles. Ce qui favorise l'assolementrotation des cultures au niveau des parcelles. Ces résultats corroborent avec ceux de Idrissou et al. (2020) qui ont montré l'influence positive de la taille du cheptel sur l'adoption par les éleveurs des stratégies d'adaptation aux changements climatiques. Aussi, les résultats sont-ils conformes à ceux de Danne et Musshoff (2017) qui ont conclu que la taille du cheptel affecte significativement la décision des agroéleveurs à participer dans les programmes de pâturage.

\section{Conclusion}

Afin de contribuer au débat scientifique sur les déterminants de l'adoption des innovations en agriculture, cette étude s'est intéressée à analyser les facteurs qui (dé)favorisent l'adoption des techniques innovantes de gestion durable de la fertilité des sols au Nord-Bénin au moyen du modèle probit multivarié. Les résultats ont révélé des taux d'adoption de $70,0 \%$ pour les mesures de la régénération du sol avec les légumineux, $68,0 \%$ pour les résidus de récoltes, $56,0 \%$ pour les fertilisants organiques (fumure et déjection animale) et de $42,0 \%$ pour l'assolement-rotation. Les résultats ont aussi révélé l'existence d'une interdépendance dans l'adoption des différentes mesures de GIFS. La décision d'adopter une mesure de GIFS est déterminée par l'adoption d'une autre mesure de GIFS et vice-versa. Dans l'ensemble, la majorité des producteurs de maïs enquêtés préfèrent adopter une combinaison de deux (32\%) ou de trois mesures de GIFS (26\%). Les résultats de l'étude ont par ailleurs révélé que la distance du village au champ du producteur, l'appartenance à un groupement, le contact avec la vulgarisation, lasuperficie en maïs, la taille du cheptel et le nombre d'actifs agricoles restent les principaux facteurs déterminants l'adoption de ces mesures de GIFS. En s'appuyant sur ces résultats empiriques, l'étude propose que les politiques agricoles visant à promouvoir l'innovation dans l'agriculture soient orientées vers l'organisation des producteurs et l'intensification du conseil agricole.

\section{CONFLIT D'INTÉRÊTS}

Les auteurs déclarent qu'il n'y a aucun conflit d'intérêts.

\section{CONTRIBUTIONS DES AUTEURS}

SAA a participé à l'élaboration du protocole, l'analyse des données, la rédaction $\mathrm{du}$ projet de manuscrit et la correction du manuscrit ; JEAC a participé à l'élaboration du protocole, la collecte de données, l'analyse des données et à la rédaction et la correction du manuscrit ; JAY a participé à la correction du manuscrit. 


\section{REMERCIEMENTS}

Les auteurs tiennent à adresser leurs remerciements à toutes les personnes impliquées dans ce travail en particulier les acteurs de la filière maïs enquêtés sur le terrain. Qu'ils en soient ici remerciés pour leur disponibilité et pour la qualité du travail.

\section{REFERENCES}

Adechian S, Sossa-Vihotogbe C, Djenontin A, Akponikpe P, Baco M. 2020. Déterminants socio-économiques et environnementaux du respect des recommandations en fertilisation minérale de quelques légumes feuilles traditionnels au Benin. Agronomie Africaine, 32(1) : 25-36.

Adegbola P, Adekambi SA. 2006. Impact économique de l'adoption de la jachère mucuna sur la pauvreté au Benin. Institut National des Recherches Agricoles du Benin. Communication à l'Atelier scientifique.

Adekambi SA, Hinnou C. 2020. Analyse de l'adoption des stratégies d'adaptation aux changements climatiques à l'aide du modèle probit multivarié. Afrique Science, 17(6) : 192 - 206.

Akakpo C, Amadji F, Sacca S, Adjé I. 1998. Essais comparatifs de dispositifs agroforestiers pour les systèmes culturaux à base de maïs et d'igname in Expérience des cultures en couloirs et de l'agroforesterie au Sud-Bénin. INRAB, Cotonou, Bénin.

Azontondé A. 1993. Dégradation et restauration des terres de barre (sols ferralitiques faiblement desaturés argilosableux) au Bénin. La gestion conservatoire de l'eau, de la biomasse et de la fertilité des sols (GCES). Cah. Orstom, Ser. Pedol., 28(2) : 217-226.

Danne M, Musshoff O. 2017. Analysis of farmers willingness to participate in pasture grazing programs: Results from a discrete choice experiment with German dairy farmers. Journal of Dairy Science, 100(9): 7569-7580. DOI: https://doi.org/10.3168/jds.2017-12756

Danso-Abbeam G, Baiyegunhi L. 2017. Adoption of agrochemical management practices among smallholder cocoa farmers in Ghana. African Journal of Science, Technology, Innovation and Development, 9(6): 717-728. DOI: https://doi.org/10.1080/20421338.2017.1 380358

Dassoundo-Assogba C, Yabi J, Pelebe R. 2019. Déterminants du choix de l'alimentation des poissons par les pisciculteurs de la vallée de l'Ouémé au Sud du Bénin. International Journal of Progressive Sciences and Technologies (IJPSAT), $\quad$ 15(1): 30-44. http://ijpsat.ijsht-journals.org

Degla P, Daanon P, Onzo A, Tomavo E. 2020. Analyse comparative des performances économiques des systèmes de production du maïs dans la commune de Banikoara au Nord-Bénin. Sciences de la Vie, de la Terre et Agronomie, $\mathbf{8}(1)$.

Etèka A. 1998. Importance des plantes de couvertures et de l'agroforesterie dans l'agriculure. Comm. 3ème Con Ord. De l'Ass. Des Ressortissants de Diho (ARDKassowokpo), 5p, Savè, Bénin.

FAO. 2015. Stimuler les sols africains. De la Déclaration d'Abuja sur les engrais au cadre de gestion durable des sols pour la sécurité alimentaire et nutritionnelle en Afrique à l'horizon 2030.16p.

Greene WH, Hensher DA. 2003. A latent class model for discrete choice analysis: contrasts with mixedlogit. Transportation Research Part B: Methodological, 37(8) : 681-698. DOI: https://doi.org/10.1016/S01912615(02)00046-2

Greene W. 2008. Econometric Analysis (6th edn). Prentice Hall, Upper Saddle River.

Idrissou Y, Assani A, Baco M, Yabi A, Traoré I. 2020. Adaptation strategies of cattle farmers in the dry and sub-humid tropical zones of Benin in the context of climate change. Heliyon, 6(7): e04373. DOI:https://doi.org/10.1016/j.heliyon.20 20.e 04373

Issoufou $\mathrm{O}$, Boubacar S, Toudou A, Boubacar Y. 2017. Modélisation des décisions des agriculteurs sur l'adoption et l'intensification des semences améliorées du niébé au Niger. Revue Marocaine des Sciences Agronomiques et Vétérinaires, 5(4) : 405-413. 
Kassie M, Teklewold H, Jaleta M, Marenya P, Erenstein O. 2015. Understanding the adoption of a portfolio of sustainable intensification practices in eastern and southern Africa. Land Use Policy, 42: 400-411.

Kini J, Pouw N, Gupta J. 2020. Organic vegetables demand in urban area using a count outcome model: case study of Burkina Faso. Agricultural and Food Economics, 8(1): 1-16.

Kpadenou C, Tama C, Tossou B, Yabi J. 2019. Déterminants socio-économiques de l'adoption des pratiques agroécologiques en production maraîchère dans la vallée du Niger au Bénin. International Journal of Biological and Chemical Sciences, 13(7) : 3103-3118. DOI:https://doi.org/10.4314/ijbcs.v13i7. 11

Mariano MJ, Villano R, Fleming E. 2012. Factors influencing farmers' adoption of modern rice technologies and good management practices in the Philippines. Agricultural Systems, 110: 41-53. DOI: https://doi.org/10.1016/j.agsy.2012.03.01 0

Oude Lansink A, van den Berg M, Huirne R. 2003. Analysis of strategic planning of Dutch pig farmers using a multivariate probit model. Agricultural Systems, 78(1): 73-84. DOI: http://dx.doi.org/10.1016/S0308521X(03)00034-9

Protection et Réhabilitation des Sols (ProSol). 2016a. Fertilité des sols au Bénin. Résultats de l'étude de fertilité des sols menée dans 4 départements au Bénin en 2016. GIZ, MAEP, FAO, WOCAT et Sol Consult Africa. 10p.

Protection et Réhabilitation des Sols (ProSol). 2016b. Mesures de Gestion Durables des Terres (GDT) : les différentes catégories de mesures de Gestion Durables des Terres et d'adaptation au changement climatique. GIZ, MAEP, INRAB. 8P.

PSDSA (Plan Stratégique de Développement du Secteur Agricole). 2017. Plan National d'Investissements Agricoles et de Sécurité Alimentaire et Nutritionnelle PNIASAN 2017 - 2021. MAEP, version finale mai 2017. $139 \mathrm{p}$.
Ramade F. 2014. Un Monde sans Famine. Vers une Agriculture Durable. Dunod : Paris.

Rogers EM. 2003. Diffusion of Innovations $\left(5^{\text {th }}\right.$ edn). Free Press: New York.

Sanou K, Soule BA. 2017. Contraintes d'adoption des technologies de gestion de la fertilité des sols en riziculture irriguée au Sud Togo. Agronomie Africaine, 29(2) : 177-184.

Shiferaw F, Tesfaye Z. 2005. Adoption of improved maize varieties in Southern Ethiopia: Factors and strategy options. Food Policy, 31: 442-457. DOI: https://doi.org/10.1016/j.foodpol.2005.12 .003

Sileshi M, Kadigi R, Mutabazi K, Sieber S. 2019. Determinants for adoption of physical soil and water conservation measures by smallholder farmers in Ethiopia. International Soil and Water Conservation Research, 7(4): 354-361. DOI:https://doi.org/10.1016/j.iswcr.2019 .08 .002

Teferi A, Philip D, Jaleta M. 2015. Factors that affect the adoption of improved maize varieties by smallholder farmers in Central Oromia, Ethiopia. Developing Country Studies, 5(15): 50-59.

Timu A, Mulwa R, Okello J, Kamau M. 2014. The role of varietal attributes on adoption of improved seed varieties: the case of sorghum in Kenya. Agriculture \& Food Security, 3(1): 9.

Varian HR. 2006. Analyse Microéconomique (6e édn). De Boeck : Bruxelles ; 824 p.

Wu J, Babcock B. 1998. The choice of tillage, rotation, and soil testing practices: Economic and environmental implications. American Journal of Agricultural Economics, 80(3): 494511.

Yabi JA, Bachabi FX, Labiyi IA, Ode CA, Ayena RL. 2016. Déterminants socioéconomiques de l'adoption des pratiques culturales de gestion de la fertilité des sols utilisées dans la commune de Ouaké au Nord-Ouest du Bénin. International Journal of Biological and Chemical Sciences, 10(2): 779-792. DOI : https://doi.org/10.4314/ijbcs.v10i2.27. 